\title{
Influencia de Niveles de Azufre en la Producción, Composición Química Bromatológica y Digestibilidad del Forraje Verde Hidropónico de Cebada (Hordeum vulgare L)
}

\author{
Effect of Sulfur Levels in the Production, Chemical Composition and \\ Digestibility of Hydroponic Green Fodder BARLey (Hordeum vulgare L)
}

A. Quispe Cusi' ${ }^{1}$, Z. Paquiyauri ${ }^{1}$, Y.V. Ramos ${ }^{1}$, J.L Contreras ${ }^{1,2}$, M.A. Véliz ${ }^{1}$

\section{Resumien}

\begin{abstract}
El presente estudio tuvo como objetivo evaluar la influencia de los niveles de azufre como aditivo en la producción, composición química bromatológica y digestibilidad del forraje verde hidropónico de la cebada. Se emplearon cuatro niveles de azufre: 0, 20, 30 y 40 ppm, y la cosecha se realizó a los 18 días. Se utilizó un diseño experimental completamente al azar con cinco repeticiones. La producción de biomasa (PB) y altura de tallo (AT) fue de $34.9 \pm 0.5 \mathrm{~kg} / \mathrm{m}^{2}$ y $14.61 \pm 1.38 \mathrm{~cm}$, respectivamente, con una respuesta lineal para $\mathrm{PB}$ de $\mathrm{Y}=5.33+0.67 \mathrm{X}$ y para AT de $\mathrm{Y}=10.10+1.80 \mathrm{X}$. El nivel $0 \mathrm{ppm}$ de $\mathrm{S}$ presentó mayores contenidos de proteína cruda (PC) y extracto libre de nitrógeno (ELN): $12.95 \pm 4.21 \%$ y $55.19 \pm 3.95 \%$, respectivamente; el nivel $30 \mathrm{ppm}$ de $\mathrm{S}$ presentó mayores contenidos de fibra cruda (FC) y fibra detergente neutro (FDN): $23.61 \pm 3.65 \%$ y $65.95 \pm 4.15 \%$, respectivamente; y el nivel de 40 ppm presentó mayores contenidos de extracto etéreo (EE) y ceniza (C): $3.88 \pm 3.15 \%$, y $4.61 \pm 2.98 \%$, respectivamente. Los valores de la digestibilidad aparente de FC y EE fueron significativamente influenciados por los niveles de azufre.
\end{abstract}

Palabras clave: consumo, proteína cruda, biomasa, solución nutritiva, cuy, forraje hidropónico

\section{Abstract}

This study aimed to evaluate the effect of sulfur levels as additive in the production, chemical composition and digestibility of hydroponic green fodder barley. Four levels of S were used: 0, 20, 30 and 40 ppm and harvest was done at 18 days. A completely random design was used with five repetitions. Biomass production (PB) and the height of stem

\footnotetext{
${ }^{1}$ Laboratorio de Nutrición Animal y Evaluación de Alimentos, Departamento Académico de Zootecnia, Facultad de Ciencias de Ingeniería, Universidad Nacional de Huancavelica, Perú

${ }^{2}$ E-mail: joselcpunh123@hotmail.com
}

Recibido: 24 de febrero de 2015

Aceptado para publicación: 18 de agosto de 2015 
(AT) were $34.9 \pm 0.5 \mathrm{~kg} / \mathrm{m}^{2}$ and $14.61 \pm 1.38 \mathrm{~cm}$, respectively, having a lineal response for PB $(\mathrm{Y}=5.33+0.67 \mathrm{X})$ and $\mathrm{AT}(\mathrm{Y}=10.10+1.80 \mathrm{X})$. The $0 \mathrm{~S}$ level showed higher crude protein $(\mathrm{CP})$ content and nitrogen-free extract: $12.95 \pm 4.21 \%$ and $55.19 \pm 3.95 \%$ respectively; the $30 \mathrm{ppm}$ level showed higher content of crude fibre $(\mathrm{CF})$ to the raw fiber and neutral detergent fiber (NDF): $23.61 \pm 3.65 \%$ and $65.95 \pm 4.15 \%$ respectively; and the $40 \mathrm{ppm}$ level showed higher content of ether extract (EE) and ash: $3.88 \pm 3.15 \%$ and $4.61 \pm 2.98 \%$, respectively. The apparent digestibility of CF and EE were significantly affected by sulfur levels.

Key words: intake, crude protein, biomass, nutrient solutions, guinea pig, hydroponic fodder

\section{INTRODUCCIÓN}

El sistema de forraje verde hidropónico puede proporcionar un suministro constante de alimento para el ganado durante épocas críticas de abastecimiento de forrajes (sequía/ invierno), siendo apropiado para su producción en áreas pequeñas de terreno (Rodríguez et al., 2005). Esta metodología ha sido incorporada en sistemas intensivos de producción animal para proporcionar un nuevo ingrediente como alimento, suplemento o reemplazo de uno o más componentes de la ración diaria (Figueroa et al., 1999; Rodríguez et al., 2005; Morales et al., 2009), debido a su alta palatabilidad, digestibilidad y por presentar, en general, niveles adecuados de energía, vitaminas y minerales (Chang et al., 2000).

El uso de la cebada (Hordeum vulgare L.) como forraje verde hidropónico en la sierra peruana ha tenido respuestas positivas en la producción de materia verde, en sus tenores químicos y en los parámetros productivos de cuyes, pudiendo llegar a sustituir hasta un $75 \%$ de la alfalfa por el germinado de cebada (Cayllahua et al., 2015).

El azufre, macroelemento esencial secundario en la nutrición de las plantas, se requiere en cantidades menores que los elementos esenciales primarios, pero es requerido para el desarrollo normal de los cultivos. El azufre, junto con el nitrógeno y el fósforo, constituyen 'elementos estructurales accesorios' para la nutrición de las plantas, y cuya función en el metabolismo es la de acompañar a los elementos estructurales principales (carbono, hidrógeno y oxígeno) para formar compuestos específicos como aminoácidos y fosfolípidos (Carrillo et al., 2010). El azufre es absorbido por la planta que lo utiliza como nutriente y favorece la actividad fotosintética de la planta; además, no solo acelera la solubilidad del cobre, sino que su presencia es esencial para la asimilación eficiente del nitrógeno, potasio y, especialmente, fósforo por la planta (Aguilera y Salazar, 1996).

En vista que el forraje verde hidropónico es un alimento de creciente utilización y el hecho de ser producido en un espacio donde los nutrientes son escasos, el presente estudio tuvo como objetivo evaluar la influencia de los niveles de azufre como fertilizante en la producción, composición química bromatológica y digestibilidad del forraje verde hidropónico de cebada.

\section{Materiales y Métodos}

\section{Lugar de Estudio}

El experimento fue conducido entre abril y julio de 2013 en la unidad experimental de producción de forrajes hidropónicos (invernadero) de la Escuela Profesional de Zootecnia de la Universidad Nacional de Huancavelica (UNH), ubicada en la provincia y departamento de Huancavelica, Perú, a una altura de $3780 \mathrm{msnm}$. 


\section{Método Experimental}

Se utilizaron cinco bandejas de $1480 \mathrm{~cm}^{2}$ por tratamiento, con una densidad de siembra al voleo de $20 \mathrm{~g}$ de semilla $/ \mathrm{dm}^{2}$, resultando en $296 \mathrm{~g}$ por bandeja. Este procedimiento se hizo debido al tamaño de la semilla y para evitar su pudrición y desarrollo de hongos.

El cultivo de forraje hidropónico de cebada (Hordeum vulgare L.) de la variedad UNA 80 se hizo con la técnica descrita por Tarrillo (2002). Se prepararon dos soluciones nutritivas. La solución A contenía 340 g de fosfato de amonio, $2.080 \mathrm{~g}$ de nitrato de calcio y $550 \mathrm{~g}$ de nitrato de potasio, en mezcla para 10 litros de agua potable. La solución B contenía $492 \mathrm{~g}$ de sulfato de magnesio, $0.48 \mathrm{~g}$ de sulfato de cobre, $2.48 \mathrm{~g}$ de sulfato de manganeso, $1.20 \mathrm{~g}$ de sulfato de zinc, $620 \mathrm{~g}$ de ácido bórico, $0.02 \mathrm{~g}$ de molibdato de amonio y $50 \mathrm{~g}$ de quelato de hierro, en mezcla para 4 litros de agua potable.

Se trabajó con la solución nutritiva comercial La Molina que contiene la solución A ( $5 \mathrm{ml} / \mathrm{L}$ de agua $)$ y la solución B $(2 \mathrm{ml} / \mathrm{L}$ de agua). Esta solución se utilizó para el riego por intermedio de aspersores a partir del $4^{\circ}$ y $7^{\circ}$ día (dos riegos por día) para todos los tratamientos. Entre el $8^{\circ}$ y $14^{\circ}$ día se realizó la aspersión del azufre elemental disuelto en agua y agregado a la solución nutritiva en los niveles de 0, 20, 30 y 40 ppm de azufre/bandeja $/ \mathrm{m}^{2}$. En el día $15^{\circ}$ se regó con agua con la finalidad de lavar el forraje de todo tipo de residuo. La cosecha se hizo en el día 18 de la siembra.

La fuente de azufre procede de procesos industriales de rocas parenterales del suelo, donde el azufre se encuentra principalmente en forma de sulfuros (sulfuro de hierro - FeS). Las concentraciones en ígneas básicas promedian $600 \mathrm{~g}$ de $\mathrm{S} / \mathrm{g}$. El azufre se oxida rápidamente a sulfato $\left(\mathrm{SO}_{4}{ }^{2-}\right)$ durante la meteorización de los sulfuros en la superficie del suelo.

\section{Variables Evaluadas}

Los parámetros de producción de biomasa (PB) y altura de tallo (AT) se determinaron en el momento de la cosecha del forraje. Asimismo, se tomaron muestras al azar para la determinación de la composición química bromatológica.

En las pruebas de digestibilidad aparente se utilizaron cinco cuyes machos por tratamiento, haciendo un total de 20 cuyes. Los animales experimentales eran de la línea Perú y tenían un mes de edad. Se les suministró el forraje verde hidropónico de cebada producido en cada tratamiento de azufre en cantidades iguales al peso vivo de los animales, pero fraccionado en una ración $(50 \%)$ en las mañanas y otra en las tardes (50\%). El alimento sobrante y las heces fueron recolectados y pesados diariamente.

La determinación de la composición química bromatológica se hizo en el Laboratorio de Bioquímica, Nutrición y Alimentación Animal de la Facultad de Medicina Veterinaria de la Universidad Nacional Mayor de San Marcos, Lima, y la digestibilidad aparente se determinó en los ambientes de la granja experimental de cuyes de la UNH.

\section{Análisis Estadístico}

Se empleó un diseño estadístico completamente al azar, con cuatro tratamientos de azufre y cinco repeticiones por tratamiento, totalizando 20 unidades experimentales. Las diferencias entre grupos fueron determinadas mediante el análisis de varianza con un nivel de significancia de $\mathrm{p}<0.05$ y un análisis de regresión. Los factores estudiados fueron los niveles de azufre en la producción de biomasa fresca, altura de tallo, composición química bromatológica y digestibilidad del forraje. Para el análisis se empleó el paquete estadístico SAS v. 9.2. 


\section{Resultados y Discusión}

En la Cuadro 1 se aprecia el efecto $(p<0.001)$ de los niveles de azufre en el forraje verde hidropónico de cebada sobre $\mathrm{PB}$ y AT, indicando que ambas variables presentan una mejora sustancial con el incremento de los niveles de azufre en la solución nutritiva. Se obtuvo una respuesta lineal positiva para PB de acuerdo a la ecuación $Y=5.33+$ 0.67X. Asimismo, la AT (Fig. 1) presentó un comportamiento lineal positivo de acuerdo a la ecuación $Y=10.10+1.80 X$.

El promedio general de $\mathrm{PB}$ en el presente estudio $\left(34.9 \pm 0.5 \mathrm{~kg} / \mathrm{m}^{2}\right)$ fue mayor a los valores de 16.8 y $19.4 \mathrm{~kg} / \mathrm{m}^{2}$ (promedio de $18.09 \mathrm{~kg} / \mathrm{m}^{2}$ ), reportados por Calles (2005), utilizando los mismos niveles de azufre en el cultivo hidropónico de cebada; en tanto que Carrasco (2005) reportó $1.6 \mathrm{~kg}$ de PB de este cultivo a los 10 días de siembra y sin el uso de azufre. Asimismo, la AT del presente estudio $(14.61 \pm 1.38 \mathrm{~cm})$ fue similar a los $16.98 \mathrm{~cm}$ obtenido por Calles (2005), pero menor a los $22.0 \mathrm{~cm}$ reportado en el trabajo de Carrasco (2005) indicado anteriormente. Por otro lado, Rojas et al. (2002) evaluaron la AT para el forraje hidropónico del centeno con dos densidades de siembra (94.2 y $282 \mathrm{~g}$ de semilla por bandeja) reportando un pro- medio general de $19.7 \mathrm{~cm}$ para 10,15 y 20 días de edad a la cosecha.

Los resultados del análisis bromatológico se presentan en el Cuadro 2. El $12.95 \%$ de proteína cruda fue mayor al $10.31 \%$ reportado por Contreras et al. (2015) con promedio de $10.31 \%$ aunque inferior al $16.26 \%$ obtenido por Calles (2005) en la producción de forraje verde hidropónico de cebada. Carrasco (2005) reportó $13.30 \%$ de proteína cruda con este cultivo a los 10 días y sin el uso de azufre. Asimismo, Rojas et al. (2002), con cultivo hidropónico de centeno obtuvo un promedio general de $18.63 \%$ de proteína cruda.

La fibra cruda presentó valores extremos de 16.95 y $27.40 \%$ ( $\mathrm{p}<0.05)$ en los niveles de 0 y $30 \mathrm{ppm} / \mathrm{m}^{2}$, respectivamente (promedio de 23.61\%). Calles (2005) reporta valores entre 23.25 y $24.52 \%$ para los niveles 30 y 40 ppm de azufre, en tanto que Carrasco (2005) reporta $20.59 \%$ sin utilizar aditivo. En relación al extracto etéreo, se obtuvo una variación de 3.55 a $4.20 \%$ para los niveles de 0 y $40 \mathrm{ppm} / \mathrm{m}^{2}(\mathrm{p}<0.05)$, valores similares al 3.35 y $4.04 \%$ para los niveles de 20 y 40 ppm empleados por Calles (2005). Asimismo, Carrasco (2005) reporta un promedio inferior $(2.63 \%)$ a los 10 días de sembrado el forraje hidropónico de cebada.

Cuadro 1. Resumen de análisis de varianza para la producción de biomasa (PB) y altura del tallo (AT) del forraje verde hidropónico de la cebada, en función a los niveles de azufre en la solución nutritiva

\begin{tabular}{lccccccc}
\hline \multirow{2}{*}{$\begin{array}{l}\text { Fuentes de } \\
\text { variación }\end{array}$} & \multirow{2}{*}{$\mathrm{GL}$} & \multicolumn{3}{c}{$\mathrm{PB}\left(\mathrm{kg} / \mathrm{m}^{2}\right)$} & \multicolumn{3}{c}{$\mathrm{AT}(\mathrm{cm})$} \\
\cline { 2 - 7 } & & $\mathrm{CM}^{\mathrm{a}}$ & $\mathrm{F}^{\mathrm{b}}$ & $\mathrm{R}(\%)^{\mathrm{c}}$ & $\mathrm{CM}^{\mathrm{a}}$ & $\mathrm{F}^{\mathrm{b}}$ & $\mathrm{R}(\%)^{\mathrm{c}}$ \\
\hline Tratamiento & 3 & 3.8 & $13.61^{*}$ & 71.84 & 28.4 & $13.52^{*}$ & 68.18 \\
Error & 16 & 0.27 & & & 2.1 & & \\
Media & & 34.9 & & & 14.61 & & \\
\hline
\end{tabular}

a: cuadrados medios; b: Prueba de F; c: relaciones entre la suma de cuadrados de los efectos y las sumas de cuadrados totales; $*$ : $p \varangle 0.001$ 

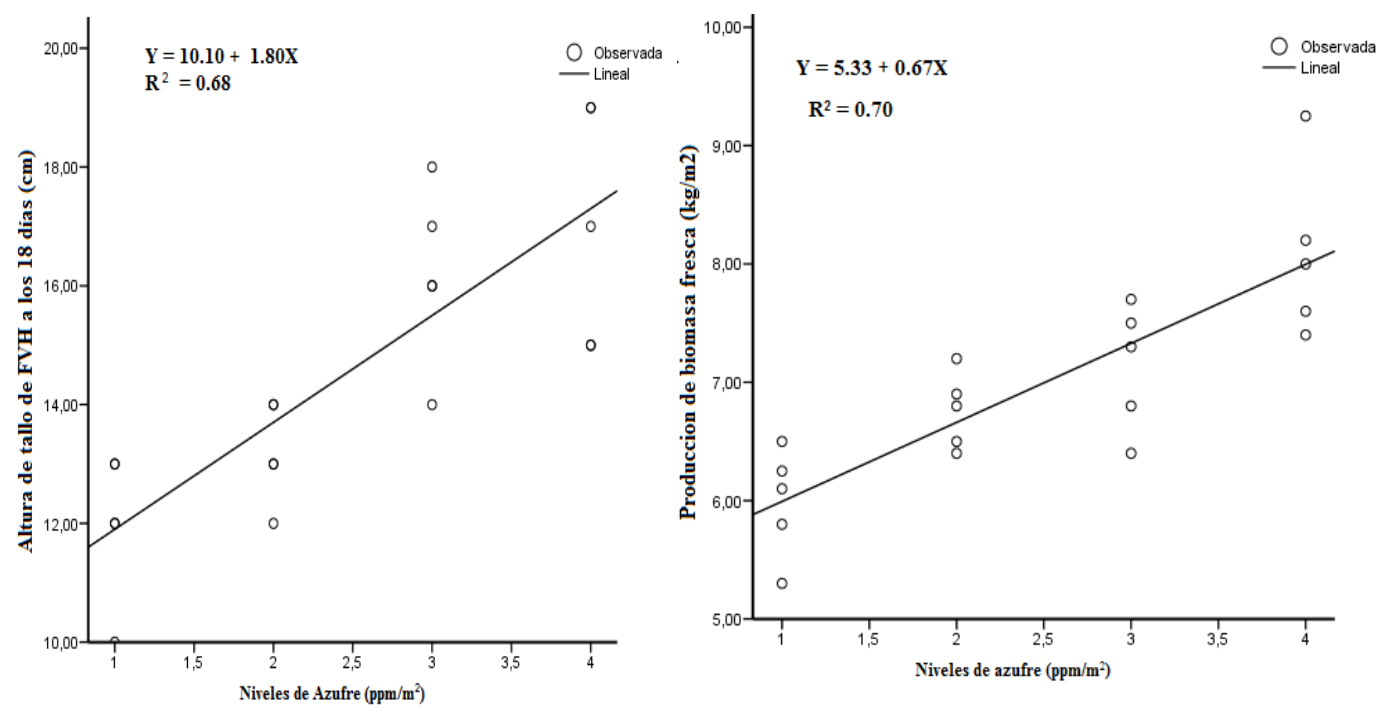

Figura 1. Curva, ecuación de regresión para la producción de biomasa $\left(\mathrm{kg} / \mathrm{m}^{2}\right)$ y altura de tallo $(\mathrm{cm})$ en función de los niveles de azufre $\left(\mathrm{ppm} / \mathrm{m}^{2}\right)$ para el forraje verde hidropónico de cebada

Cuadro 2. Análisis químico bromatológico del forraje verde hidropónico de la cebada

\begin{tabular}{cccccccc}
\hline $\begin{array}{c}\text { Niveles } \\
\text { de azufre } \\
\left(\mathrm{ppm} / \mathrm{m}^{2}\right)\end{array}$ & $\begin{array}{c}\text { MS } \\
(\%)\end{array}$ & $\begin{array}{c}\text { PC } \\
(\%)\end{array}$ & $\begin{array}{c}\text { FC } \\
(\%)\end{array}$ & $\begin{array}{c}\text { EE } \\
(\%)\end{array}$ & $\begin{array}{c}\text { ELN } \\
(\%)\end{array}$ & $\begin{array}{c}\text { C } \\
(\%)\end{array}$ & $\begin{array}{c}\text { FDN } \\
(\%)\end{array}$ \\
\hline 0 & $16.22^{\mathrm{b}}$ & $14.78^{\mathrm{b}}$ & $16.95^{\mathrm{b}}$ & $3.55^{\mathrm{b}}$ & $60.30^{\mathrm{b}}$ & $4.42^{\mathrm{b}}$ & $63.68^{\mathrm{b}}$ \\
20 & $16.37^{\mathrm{a}}$ & $11.78^{\mathrm{a}}$ & $25.69^{\mathrm{a}}$ & $3.61^{\mathrm{a}}$ & $54.48^{\mathrm{a}}$ & $4.44^{\mathrm{b}}$ & $67.32^{\mathrm{a}}$ \\
30 & $15.99^{\mathrm{c}}$ & $12.04^{\mathrm{c}}$ & $27.40^{\mathrm{c}}$ & $4.16^{\mathrm{c}}$ & $52.86^{\mathrm{c}}$ & $4.54^{\mathrm{b}}$ & $68.66^{\mathrm{c}}$ \\
40 & $15.92^{\mathrm{d}}$ & $13.22^{\mathrm{d}}$ & $24.42^{\mathrm{d}}$ & $4.20^{\mathrm{c}}$ & $53.12^{\mathrm{d}}$ & $5.04^{\mathrm{a}}$ & $64.43^{\mathrm{d}}$ \\
\hline Promedio & 16.12 & 12.95 & 23.61 & 3.88 & 55.19 & 4.61 & 65.95 \\
\hline
\end{tabular}

MS: materia seca; PC: proteína cruda; FC: fibra cruda; EE: extracto etéreo; ELN: extracto libre de nitrógeno; C: ceniza; FDN: fibra detergente neutro

${ }^{a, b}$ Superíndices diferentes dentro de columnasindican diferencia estadística $(p \varangle 0.05)$

El extracto libre de nitrógeno presentó un valor promedio de $55.19 \%$ y sin diferencias estadísticas entre niveles de azufre, no habiéndose encontrado datos comparativos en la literatura. El promedio de contenido de ceniza fue de $4.61 \%$, valor inferior a los valores de 5.4 y $5.3 \%$ reportados por Rojas et al. (2002), quienes evaluaron el cultivo hidropónico del centeno con densidades de siembra de 94 a $282 \mathrm{~g}$ de semilla por bandeja y con edades de cosecha entre 10 y 20 días. Por otro lado, el valor promedio para la fibra detergente neutro fue de $65.95 \%$, valor superior al $48 \%$ obtenido por Rojas et al. (2002) con cultivo hidropónico del centeno. 
Cuadro 3. Resumen de análisis de varianza de la digestibilidad aparente de proteína cruda (DAPC), fibra cruda (DAFC), extracto etéreo (DAEE), extracto libre de nitrógeno (DAELN), y nutrientes digestibles totales (\% NDT) del forraje verde hidropónico de la cebada, en función a los niveles de azufre

\begin{tabular}{|c|c|c|c|c|c|c|c|c|c|c|c|c|c|c|c|c|}
\hline \multirow{2}{*}{$\begin{array}{l}\text { Fuente } \\
\text { de } \\
\text { variación }\end{array}$} & \multirow[b]{2}{*}{ GL } & \multicolumn{3}{|c|}{ DAPC $(\%)$} & \multicolumn{3}{|c|}{$\operatorname{DAFC}(\%)$} & \multicolumn{3}{|c|}{ DAEE $(\%)$} & \multicolumn{3}{|c|}{ DAELN (\%) } & \multicolumn{3}{|c|}{ NDT $(\%)$} \\
\hline & & $\mathrm{CM}^{\mathrm{a}}$ & $\mathrm{F}^{\mathrm{b}}$ & $\begin{array}{l}\mathrm{RC}^{\mathrm{c}} \\
(\%)\end{array}$ & $\mathrm{CM}$ & F & $\begin{array}{l}\mathrm{RC} \\
(\%)\end{array}$ & $\mathrm{CM}$ & $\mathrm{F}$ & $\begin{array}{l}\mathrm{RC} \\
(\%)\end{array}$ & $\mathrm{CM}$ & $\mathrm{F}$ & $\begin{array}{l}\mathrm{RC} \\
(\%)\end{array}$ & $\mathrm{CM}$ & F & $\begin{array}{l}\mathrm{RC} \\
(\%)\end{array}$ \\
\hline Tratamiento & 3 & 31 & $1.8^{1}$ & 24.9 & 184 & $9.7^{3}$ & 64.5 & 20.8 & $4.1^{2}$ & 43.5 & 34.9 & $2.3^{1}$ & 30.3 & 6.3 & $0.4^{1}$ & 7.4 \\
\hline Error & 16 & 17.5 & & & 19.0 & & & 5.1 & & & 15.1 & & & 14.7 & & \\
\hline CV (\%) & & 5.6 & & & 6.2 & & & 2.6 & & & 5.2 & & & 5.1 & & \\
\hline Media & & 75.3 & & & 70.8 & & & 84.7 & & & 75.1 & & & 75.4 & & \\
\hline
\end{tabular}

a: cuadrados medios; b: Prueba de F; c: relaciones entre la suma de cuadrados de los efectos y las sumas de cuadrados totales; $*: p<0.001$

${ }^{1}$ No significativo; $2: p \varangle 0.05 ;^{3}: p<=001$

Cuadro 4. Valores medios y desviaciones estándar (s.d.) de los efectos de los niveles de azufre sobre la digestibilidad aparente de proteína cruda (PC), fibra cruda (FC), extracto etéreo (EE), extracto libre de nitrógeno (ELN) y nutrientes digestibles totales (NDT) del forraje verde hidropónico de la cebada

\begin{tabular}{cccccc}
\hline $\begin{array}{c}\text { Niveles de } \\
\text { azufre } \\
\left(\mathrm{ppm} / \mathrm{m}^{2}\right)\end{array}$ & $\mathrm{PC}$ & $\mathrm{FC}$ & $\mathrm{EE}$ & $\begin{array}{c}\text { NDT } \\
(\%)\end{array}$ \\
\cline { 2 - 5 } & $\overline{\mathrm{X}} \pm$ s.d. & $\overline{\mathrm{X}} \pm$ s.d. & $\overline{\mathrm{X}} \pm$ s.d. & $\overline{\mathrm{X}} \pm$ s.d. & $\overline{\mathrm{X}} \pm$ s.d. \\
\hline 0 & $77.50 \pm 4.46^{\mathrm{b}}$ & $82.97 \pm 1.94^{\mathrm{b}}$ & $62.38 \pm 4.29^{\mathrm{a}}$ & $78.29 \pm 2.48^{\mathrm{c}}$ & $75.86 \pm 2.93^{\mathrm{b}}$ \\
20 & $73.08 \pm 3.38^{\mathrm{a}}$ & $83.16 \pm 2.11^{\mathrm{a}}$ & $70.79 \pm 3.66^{\mathrm{b}}$ & $74.48 \pm 3.20^{\mathrm{a}}$ & $74.13 \pm 3.25^{\mathrm{a}}$ \\
30 & $73.11 \pm 3.49^{\mathrm{c}}$ & $85.22 \pm 1.92^{\mathrm{c}}$ & $76.15 \pm 3.09^{\mathrm{c}}$ & $71.91 \pm 3.64^{\mathrm{b}}$ & $74.65 \pm 3.37^{\mathrm{a}}$ \\
40 & $77.31 \pm 5.14^{\mathrm{b}}$ & $87.31 \pm 2.88^{\mathrm{d}}$ & $74.08 \pm 5.88^{\mathrm{d}}$ & $75.57 \pm 5.54^{\mathrm{d}}$ & $76.71 \pm 5.33^{\mathrm{d}}$ \\
\hline Promedio & $75.25 \pm 4.11$ & $84.66 \pm 2.21$ & $70.85 \pm 4.21$ & $75.06 \pm 3.71$ & $75.33 \pm 3.72$ \\
\hline
\end{tabular}

${ }^{a, b}$ Superíndices diferentes dentro de columnasindican diferencia estadística $(p \varangle 0.05)$

En el Cuadro 3 se muestran los promedios del análisis de varianza de la digestibilidad aparente de la proteína cruda, fibra cruda, extracto etéreo, extracto libre de nitrógeno y nutrientes digestibles totales, observándose diferencia estadística por el efecto de los niveles de azufre para el extracto etéreo $(\mathrm{p}<0.05)$ y la fibra cruda $(\mathrm{p}<0.001)$. La va- riación biológica por efecto de los niveles de azufre se presentan en el Cuadro 4. No se encontraron trabajos donde se haya determinado digestibilidad del forraje hidropónico de cebada; sin embargo, Herrera et al. (2007) determinaron la digestibilidad aparente del forraje hidropónico de maíz en ovinos estabulados en jaulas metabólicas obtenien- 
Cuadro 5. Ecuaciones de regresión ajustadas para la digestibilidad aparente de la proteína cruda (DAPT), fibra cruda (DAFC), extracto etéreo (DAEE) y extracto libre de nitrógeno (DAELN) del forraje verde hidropónico de la cebada, en función al nivel de azufre

\begin{tabular}{llcc}
\hline Variable & \multicolumn{1}{c}{ Regresión } & $\mathrm{R}^{2}$ & Significancia \\
\hline DAPT $(\%)$ & $\mathrm{Y}=86.16-10.83 \mathrm{X}+2.15 \mathrm{X}^{2}$ & 0.24 & 0.05 \\
DAFC $(\%)$ & $\mathrm{Y}=47.65+17.13 \mathrm{X}-2.62 \mathrm{X}^{2}$ & 0.63 & 0.01 \\
DAEE $(\%)$ & $\mathrm{Y}=80.89+1.51 \mathrm{X}$ & 0.39 & 0.01 \\
DAELN $(\%)$ & $\mathrm{Y}=87.08-10.41 \mathrm{X}+1.87 \mathrm{X}^{2}$ & 0.28 & 0.05 \\
\hline
\end{tabular}

do una digestibilidad aparente de la materia seca de 55.9.

En el Cuadro 5 se muestra una respuesta cuadrática para la digestibilidad aparente de la proteína cruda, de la fibra cruda y del extracto libre de nitrógeno, en tanto que se observó una respuesta lineal positiva para la digestibilidad aparente de extracto etéreo del forraje hidropónico de cebada.

\section{Conclusiones}

Los niveles de azufre en la solución nutritiva influencian la producción de biomasa y altura de tallo del forraje verde hidropónico de la cebada.

\section{Agradecimientos}

Los autores agradecen de manera especial a la Granja Experimental de Animales Menores del Centro de Producción y Desarrollo de la Facultad de Ciencias de Ingeniería de la Universidad Nacional de Huancavelica por las facilidades prestadas y el apoyo con animales. Asimismo, se agradece las valiosas sugerencias y aporte en la revisión del presente artículo a las ingenieras zootecnistas Quispe Cusi y Zoraida Paquiyauri Lizana.

\section{Literatura Citada}

1. Aguilera JL, Salazar S. 1996. Efecto del nitrógeno, fósforo y potasio en el rendimiento y tamaño del fruto de aguacate. Folleto Técnico N. ${ }^{\circ}$ 12. Campo Experimental Uruapan: INIFAP. 65 p.

2. Calles D. 2005. Evaluación de la producción y calidad del forraje verde hidropónico (FVH) de cebada, con la utilización de diferentes niveles de azufre y su respuesta en ganado lechero. Tesis de Ingeniero Zootecnista. Riobamba, Ecuador: Escuela Superior Politécnica de Chimborazo. $130 \mathrm{p}$.

3. Carrillo C, Ruiz M, Aular L, Mora R, Castillo L, Arrieche I, Díaz T, et al. 2010. Un método turbidimétrico para determinar azufre en fertilizantes inorgánicos. Venesuelos 18: 6-15.

4. Carrasco JI. 1994. Utilización de la cebada (Hordeum vulgare) germinada en la alimentación de cuyes machos en crecimiento y engorde. Tesis de Ingeniero Zootecnista. Lima: Univ Nacional Agraria la Molina. $64 \mathrm{p}$.

5. Cayllahua CF, Condori DD, Cordero AF, Veliz LM, Contreras PJ. 2015. Sustitución gradual de la alfalfa (Medicago sativa L.) por el germinado de cebada (Hordeum vulgare) en raciones de cuyes (Cavia porcellus L.) en la etapa 
de crecimiento. Rev Complutense Cienc Vet 9(2): 7-21.

6. Contreras JL, Tunque M, Cordero AF. 2015. Rendimiento hidropónico de la arveja con cebada y trigo en la producción de germinados. Rev Inv Vet Perú 26: 919. doi: 10.15381/rivep.v26i1.10910

7. Chang M, Hoyos M, Rodríguez A. 2000. Producción de forraje verde hidropónico. Centro de Investigación de Hidroponía y Nutrición Mineral. Lima, Perú. [Internet]. Disponible en: http:// ww w. omarbols o 60 . com.u y/ forraje\%20(1).pdf

8. Figueroa $M$, Bórquez $F$, Tima $M$, Leighton P. 1999. Condiciones ambientales y uso de solución nitrogenada en producción de forraje «hidropónico» de avena (Avena sativa L.) en invernadero. Rev Agroci 15(2): 195-206.

9. Herrera AM, Depablos Alviárez LA, López RL. 2007. Degradabilidad y digestibilidad de la materia seca del forraje hidropónico de maíz (Zea mays). Rev Cient (Maracaibo) 17: 372-379.
10. Morales M, Fuente B, Juárez M, Ávila E. 2009. Effect of substituting hydroponic green barley forage for a commercial feed on performance of growing rabbits. World Rabbit Sci 17: 3538. doi: 10.4995/wrs.2009.668

11. Rodríguez Muela C, Rodríguez $\mathrm{HE}$, Ruiz O, Flores A, Grado JA, Arzola C. 2005. Use of green fodder produced in hydroponics systems as supplement for salers lactating cows during the dry season. Proc W S Am Soc Anim Sci 56: 271-274.

12. Rojas M, Del Carpio PA, Guerrero J. 2002. Cultivo hidropónico de centeno forrajero: densidad, edad de utilización y respuesta en cuyes criollos en crecimiento. En: XXII Reunión Científica APPA. Lambayeque, Perú: Asociación Peruana de Producción Animal.

13. Tarrillo H. 2002. Producción de forraje verde hidropónico en Arequipa, Perú. Bol Informativo de Red Hidroponía. Lima: UNALM. 45 p. 